\title{
Biomimetic Design of a Single DOF Stephenson III Leg Mechanism
}

\author{
Wafa Batayneh ${ }^{1}$, Omar Al-Araidah ${ }^{2} \&$ Salaheddin Malkawi ${ }^{3}$ \\ ${ }^{1}$ Mechanical Engineering Department, Jordan University of Science and Technology, Irbid, Jordan \\ ${ }^{2}$ Industrial Engineering Department, Jordan University of Science and Technology, Irbid, Jordan \\ ${ }^{3}$ Nuclear Engineering Department, Jordan University of Science and Technology, Irbid, Jordan \\ Correspondence: Wafa Batayneh, Mechanical Engineering Department, Jordan University of Science and \\ Technology, Irbid 22110, Jordan. Fax: 962-2-720-1074. Tel: 962-2-720-1000 ext.22692. E-mail: \\ batayw@just.edu.jo
}

Received: May 9, 2013

doi:10.5539/mer.v3n2p43
Accepted: June 9, 2013 Online Published: July 17, 2013

URL: http://dx.doi.org/10.5539/mer.v3n2p43

\begin{abstract}
The article presents a single degree-of-freedom (DOF) Stephenson III mechanical reproduction of the shape and movement of the human leg. Type and motion synthesis of the mechanism are achieved through qualitative and interactive design heuristics. Qualitative considerations include the slim straight shape of the human leg to help keeping the center of mass of the body far from ground. Moreover, the driving force and controllers of the walking apparatus are located within the upper part of the leg. We utilize Working Model software package to test and tune the segments of the mechanism to arrive at the desired walking gait. The resulting mechanism is a reproduction of the human walking apparatus with minimum control.
\end{abstract}

Keywords: Stephenson III, leg mechanism, walking gait

\section{Introduction}

Human, like other animals, utilize their walking apparatus to help satisfy their needs that require relocation. The special features of the shape of the human leg help keeping the center of mass of the body far from ground where the driving force and controllers of the walking apparatus are also located. In this article, we give special focus to the shape of the leg to preserve the advantages of the human walking apparatus. To this end, the article presents a single degree-of-freedom (DOF) Stephenson III mechanical reproduction of the shape and movement of the human leg. Type and motion synthesis of the mechanism are achieved through qualitative and interactive design heuristics. We utilize Working Model software package to test and tune the segments of the mechanism to arrive at the desired walking gait. The resulting mechanism is a reproduction of the human walking apparatus with minimum control.

Legs and the control thereof are the most significant elements for accomplishing displacement needs of a human. Building on the fact that the human walking gait is repeatable and measurable, many researchers proposed openand closed-loop mechanical reproductions of the human walking apparatus. Such mechanisms found many applications ranging from toys to space exploration. Open-loop kinematic chains have multiple degrees of freedom that make them generally flexible and easier to design. On the other hand, the many actuators used to operate the open loop mechanism adds to the complexity of the controller and hence the cost of the machine. On the contrary, closed-loop chains are difficult to design and easier to control utilizing mostly a single degree of freedom.

In the literature, Weber W. and Weber E. (1991) observed the human walking apparatus and showed that the skeletal system constitutes a mechanism that can potentially be reproduced mechanically. Jun and Krovi (2004) examined and evaluated a two DOF four leg-wheel subsystem. In (Collins \& Ruina, 2005), the authors presented a five DOF design for a three dimensional bipedal walking robot with human-like morphology and gait. Yavin (2002) considered the modeling and control of a three DOF walking four-bar open chain linkage robot. Sangwan et al. (2004) introduced a design for a two-leg robot with legs connected in series at the hip joint through a motor. In addition, a passive knee joint that has a knee stopper restricting the forward motion has been added to the mechanism. In (Verrelst et al., 2004), the authors designed a biped walking mechanism actuated using pneumatic muscles. Capi (2002) introduced an optimal scheme for a biped robot that has two legs with two DOF each. One translational DOF achieved by a DC motor attached at the body of the robot, and the other DOF is the rotational 
motion at the ankle joint. In (Mehdigholi \& Akbarnejad, 2008), the authors applied a Genetic Algorithm (GA) optimization scheme to Watt six bar leg mechanism. The mechanism consists of a four bar linkage integrated to its cognate. The design generates a straight and parallel motion path similar to the human walking gait. Batayneh et al. (2008) presented a single DOF Watt I six-bar mechanism that typifies the human's motion. The proposed mechanism includes a hip, femur, knee, and fibula. Companies such as Honda Motors, Kawada Industries and Sony played a major roll in the competition towards developing state of the art legged robots, see (Hirai et al., 1998; Hirose et al., 2001; Kaneko et al., 2004; Kuroki et al., 2001; Hirukawa et al., 2004). The proposed designs generally focus on creating the desired walking gait while overlooking the shape of the leg. Among the many linkages proposed in the literature, six-bar mechanisms show promising results combining the looks and the function of the leg mechanism. This article utilizes observations of previous works in designing a closed-loop Stephenson III human-like leg mechanism.

The walking pace of the human walking apparatus can be divided into two main phases including the flying phase and the contact phase. During the flying phase, the foot loses its contact with the terrain to relocate forward or backward. During this phase, the torque required by the driving crank is a function of the displacements and the masses of the leg segments. Once in touch with the terrain (the contact phase), the foot assumes a fixed position while the center of mass of the machine moves a horizontal distance equal to the step size generated in the first phase. During this phase, the foot is subjected to external forces from the ground that requires added torque by the motor to prevent the mechanism from collapsing. Forward and backward motions can be achieved by controlling the direction of rotation of the crank link. While the flying phase may assume various arched contours, the contact phase assumes a straight-line to insure no slip. Figure 1 shows alternative shapes of acceptable human-like walking gaits.
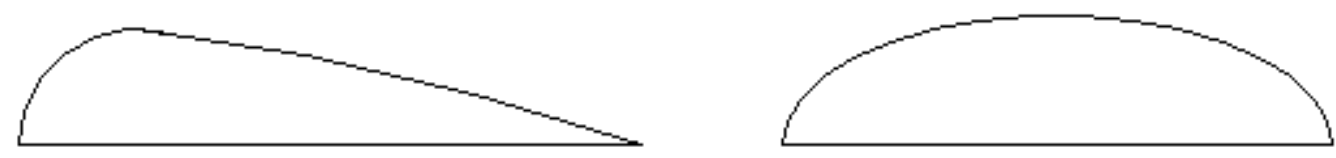

Figure 1. Alternative shapes of the human walking gait

The rest of the paper is organized as follows. Section 2 describes procedures adapted for designing the proposed leg mechanism. Section 3 addresses the position analysis of the proposed linkage. In section 4, we test the proposed model using the Working Model software package. Section 5 summarizes the work and offers concluding remarks.

\section{Qualitative Synthesis and Mechanism Description}

In looking for a suitable design, several design concepts are generated based on the following design requirements. First, the mechanism is a closed loop single DOF chain. Second, the shape of the linkage emulates the slim shape of the human leg. Furthermore, the mechanism must include a hip joint, a thigh, a knee joint, a cnemis, and an ankle joint. Third, the mechanism generates a walking gait similar to that of a human walking apparatus. Forth, the driving rotary input is located at the upper part of the leg close to the hip joint. To this end, we present a two-stage procedure to shape, test, and tune the segments of the mechanism.

To create a human-like leg mechanism, we fashioned a four bar trapezoid chain to represent the thigh. While the shortest link represents the knee side of the thigh, the opposite second shortest link represent the hip side where one end of the hip link is connected to the ground to resemble the hip joint. Moreover, the shortest link is extended to form the cnemis as illustrated in Figure 2. To test the usability of the design, we utilize Working Model software package to fashion a cam-follower mechanism, as illustrated in Figure 3, in the shape of the required walking gait. The linkage is then attached to the follower at the foot joint. By allowing the follower to rotate around its pivoting center, the foot joint follows the contour of the cam. Results show that the general motion of the resulting mechanism resembles that of the human leg during walking. Therefore, efforts are focused on allocating the location and length of the driving crank to generating an acceptable walking path. To this end, the motion paths of several points at the long link opposite to the hip joint are traced. We limit the search to that link since the motions of the other links are limited to oscillation around the hip joint. As a result, a crank is connected to that point while the foot joint is disconnected from the follower and the resulting gait is then traced. Figure 4 shows the resulting gait compared to the shape of the cam. 


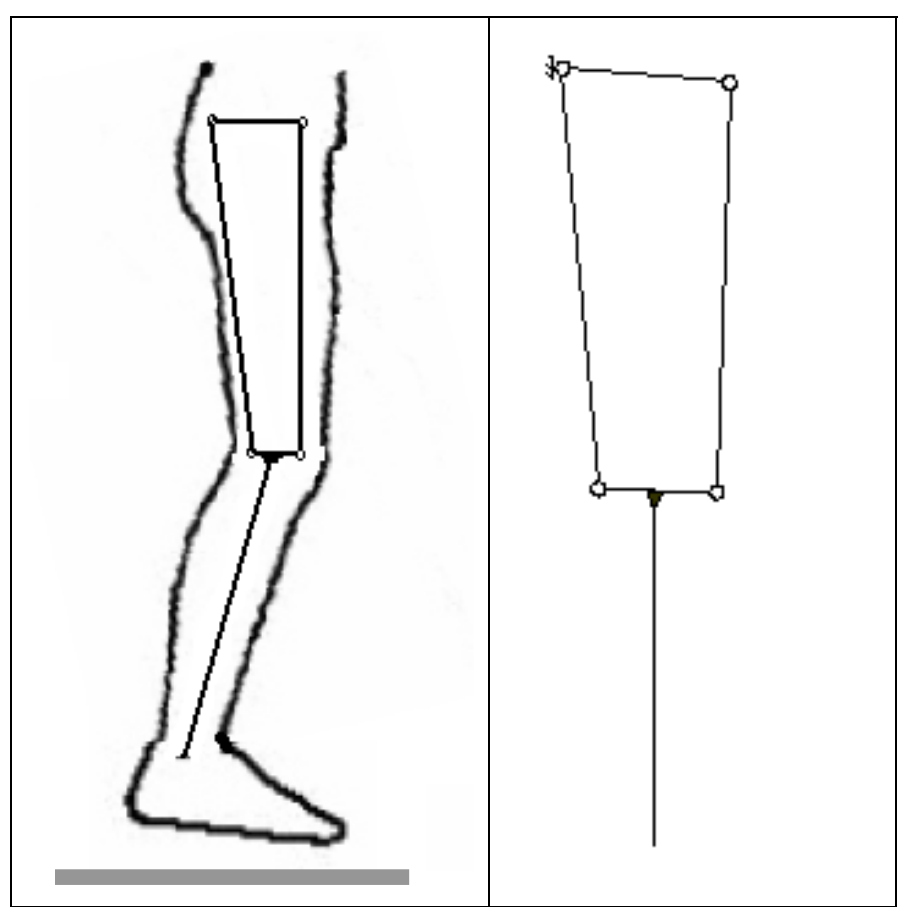

Figure 2. The building blocks of the proposed leg mechanism

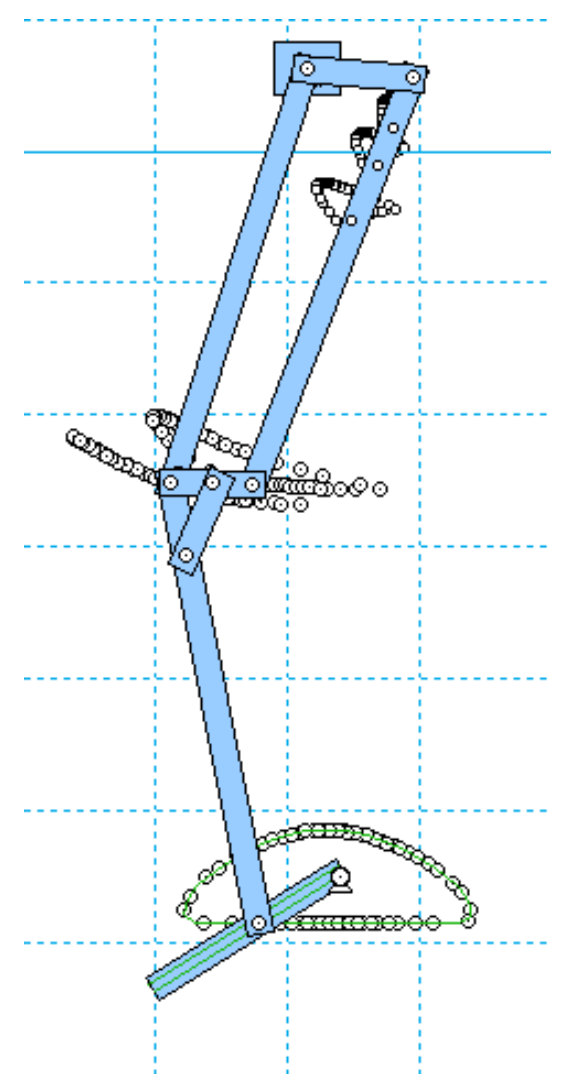

Figure 3. Usability test model for the proposed mechanism 


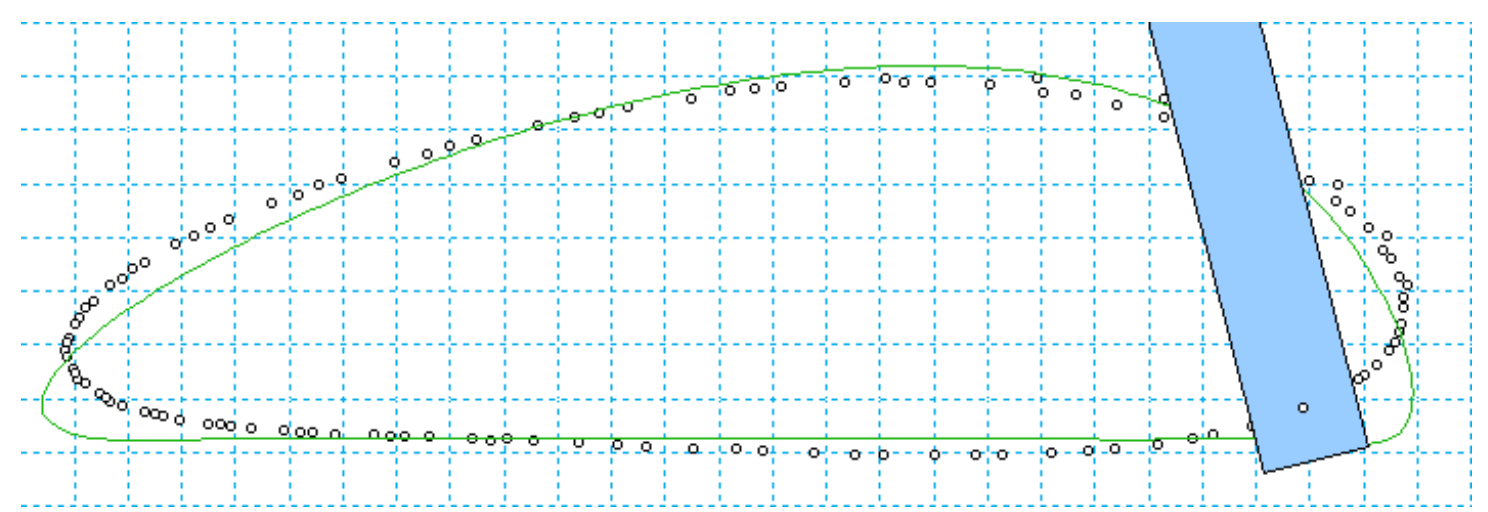

Figure 4 . The resulting walking gait compared to the shape of the cam

Consulting literature, the resulting mechanism is a Stephenson III single DOF six-bar mechanism. Figure 5 illustrates a refined shape of the mechanism. The mechanism resembles the shape of a human leg. Furthermore, the support joints and the driver are located at the upper part of the mechanism that places the body of the walking machine far from the ground.

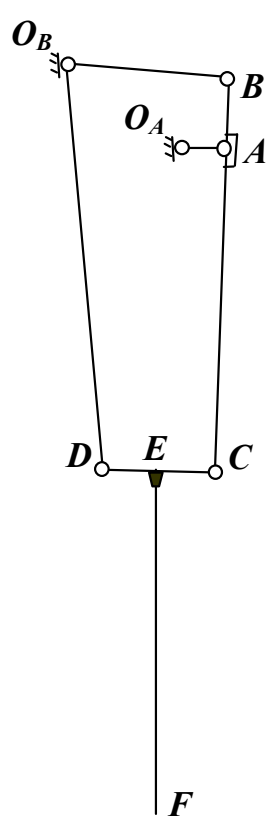

Figure 5. The proposed human-like leg mechanism

As illustrated in Figure 5, the mechanism is composed of the crank-rocker four-bar linkage $\boldsymbol{O}_{A} \boldsymbol{A} \boldsymbol{O}_{\boldsymbol{B}} \boldsymbol{B}$ with coupler point located at $\boldsymbol{C}$, and the dyad $\boldsymbol{O}_{\boldsymbol{B}} \boldsymbol{D} \boldsymbol{C}$ with a coupler point located at the foot-point $\boldsymbol{F}$. The rotation of the crank $\boldsymbol{O}_{\boldsymbol{A}} \boldsymbol{A}$ generates a foot-point path for normal walking. The link proportions of the mechanism are such that the path generated at point $\boldsymbol{F}$ emulates that used by human (see figure 1). Section 3 presents the position analysis of the proposed linkage to understand the impact of the various design parameters on the performance of the mechanism.

\section{Position Analysis of the Mechanism}

The six-bar mechanism illustrated in Figure 5 is a planar single DOF mechanism. Let joint $\boldsymbol{O}_{\boldsymbol{B}}$ represent the reference point for the mechanism. The $\boldsymbol{X}$ - and $\boldsymbol{Y}$-coordinates of the foot-point are derived using simple geometric analysis. Figure 6 presents the kinematic graph representation of the mechanism. The graph shows two independent loops, seven rotational joints, and hence one degree-of-freedom. The position analysis is carried out next. 


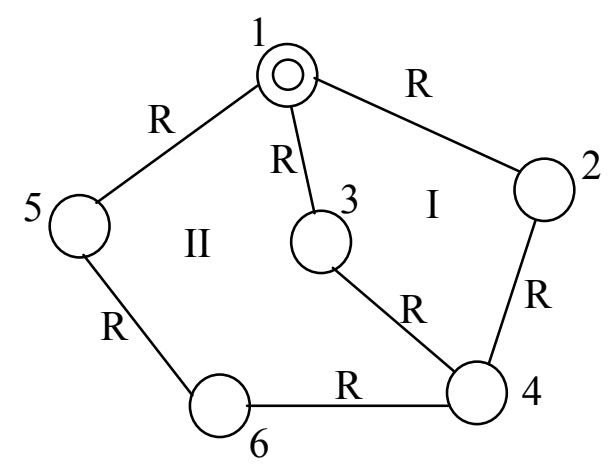

Figure 6. The kinematic graph for the leg mechanism

Two transmission angles are identified in the mechanism namely $\angle \boldsymbol{O}_{B} \boldsymbol{B} \boldsymbol{A}$ or $\gamma_{1}$ and $\angle \boldsymbol{O}_{B} \boldsymbol{D} \boldsymbol{C}$ or $\gamma_{2}$. Figure 7 shows the two transmission angles at a specific crank angle $\alpha$. Values for the transmission angles can be obtained for any given values of $\angle \boldsymbol{O}_{B} \boldsymbol{O}_{\boldsymbol{A}} \boldsymbol{A}$ or $\boldsymbol{\alpha}$ as illustrated in Equations 1 and 2 below. For better transmission of force, a transmission angle is kept around 90 degrees.
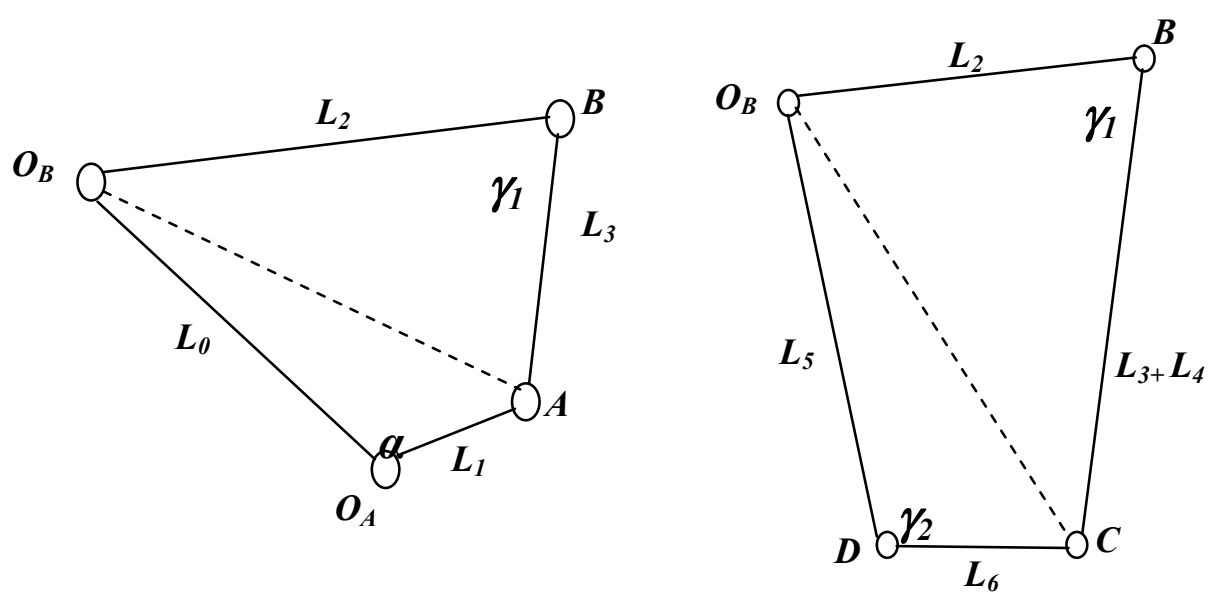

Figure 7. Transmission angles of the mechanism

$$
\cos \gamma_{1}=\frac{L_{2}^{2}+L_{3}^{2}-\left(L_{0}^{2}+L_{1}^{2}\right)}{2 L_{2} L_{3}}+\frac{L_{0} L_{1}}{L_{2} L_{3}} \cos \alpha
$$

and

$$
\cos \gamma_{2}=\frac{L_{5}^{2}+L_{6}^{2}-L_{2}^{2}-\left(L_{3}+L_{4}\right)^{2}}{2 L_{5} L_{6}}+\frac{L_{2}\left(L_{3}+L_{4}\right)}{L_{5} L_{6}} \cos \gamma_{1}
$$

The coordinates of point $\boldsymbol{F}$ are synthesized through the composition of the three closed loops $\boldsymbol{O}_{A} \boldsymbol{A C F}, \boldsymbol{O}_{\boldsymbol{B}} \boldsymbol{B C F}$, and $\boldsymbol{O}_{B} \boldsymbol{D C F}$. Denote by $\boldsymbol{L}_{\boldsymbol{\delta}}$ the length of link segment $\boldsymbol{C E}$ and by $\boldsymbol{L}_{9}$ the length of link segment $\boldsymbol{E F}$ in Figure 5, the $\boldsymbol{X}$ - and $\boldsymbol{Y}$-coordinates of point $\boldsymbol{F}$ are given by the composition of that of points $\boldsymbol{C}$ and $\boldsymbol{D}$ such that

$$
\begin{gathered}
X_{F}=X_{C}-\frac{L_{8}}{L_{6}}\left(X_{C}-X_{D}\right)-\frac{L_{9}}{L_{6}}\left(Y_{C}-Y_{D}\right) \\
Y_{F}=Y_{C}-\frac{L_{8}}{L_{6}}\left(Y_{C}-Y_{D}\right)+\frac{L_{9}}{L_{6}}\left(X_{C}-X_{D}\right)
\end{gathered}
$$

where, 


$$
\begin{gathered}
X_{C}^{2}+Y_{C}^{2}=L_{5}^{2}+L_{6}^{2}-2 L_{5} L_{6} \cos \gamma_{2} \\
X_{C}^{2}+Y_{C}^{2}=L_{2}^{2}+\left(L_{3}+L_{4}\right)^{2}-2 L_{2}\left(L_{3}+L_{4}\right) \cos \gamma_{1} \\
\left(X_{C}-X_{D}\right)^{2}+\left(Y_{C}-Y_{D}\right)^{2}=L_{6}^{2}
\end{gathered}
$$

and

$$
X_{D}^{2}+Y_{D}^{2}=L_{5}^{2}
$$

Point $\boldsymbol{F}$ follows a straight line for the contact part of its generated path. Hence, we select the lengths of the segments such that the deviation of the traced path from the straight line is minimized.

Next section addresses results obtained from iteratively resizing the lengths of the segments of the mechanism aiming at creating the human walking gait.

\section{Experimental Validation}

To validate the function ability of the proposed mechanism, we utilize Working Model software package to model, test, and modify the mechanism. We generate and test various versions of the mechanism with different link proportions. The shape of the mechanism is such that the length of the mechanism is far larger than its width. Figure 8 illustrates a sample of the generated mechanisms including the generated walking gait and motion graphs of various points on the linkages. An alternative design is illustrated in Figure 9. The designs are created such that the length of the thigh portion of the leg equals that of the cnemis. The proposed alternatives illustrate the fact that the mechanism can be optimized over two integrated stages. These include generating a four bar to generate the inverted walking gait, and the design of the dyad to invert and magnify the gait.

From Figure 8, notice that the position of the foot point influences largely the resulting footpath. Alternative foot points and hence footpaths are presented in the Figure 9. Notice the change on the shape of the path as the distance of the foot point from the knee joint increases. The mechanism in Figure 9 is rather shorter and wider than that in Figure 8. Moreover, the cnemis stars at point $\boldsymbol{D}$ rather than the midpoint of segment $\boldsymbol{C D}$.

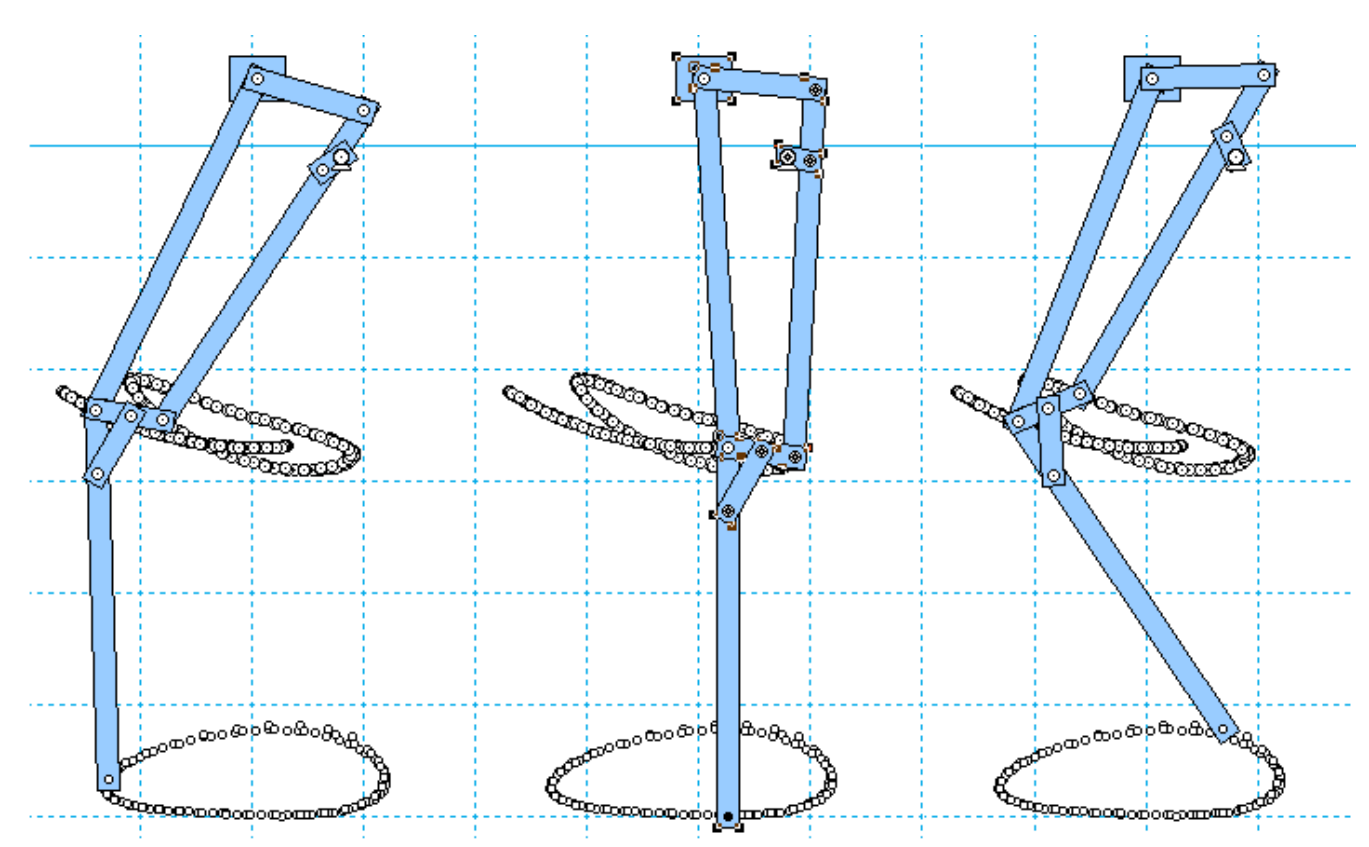

Figure 8. Sample design of the proposed human-like leg mechanism created and tested using Working Model software package 


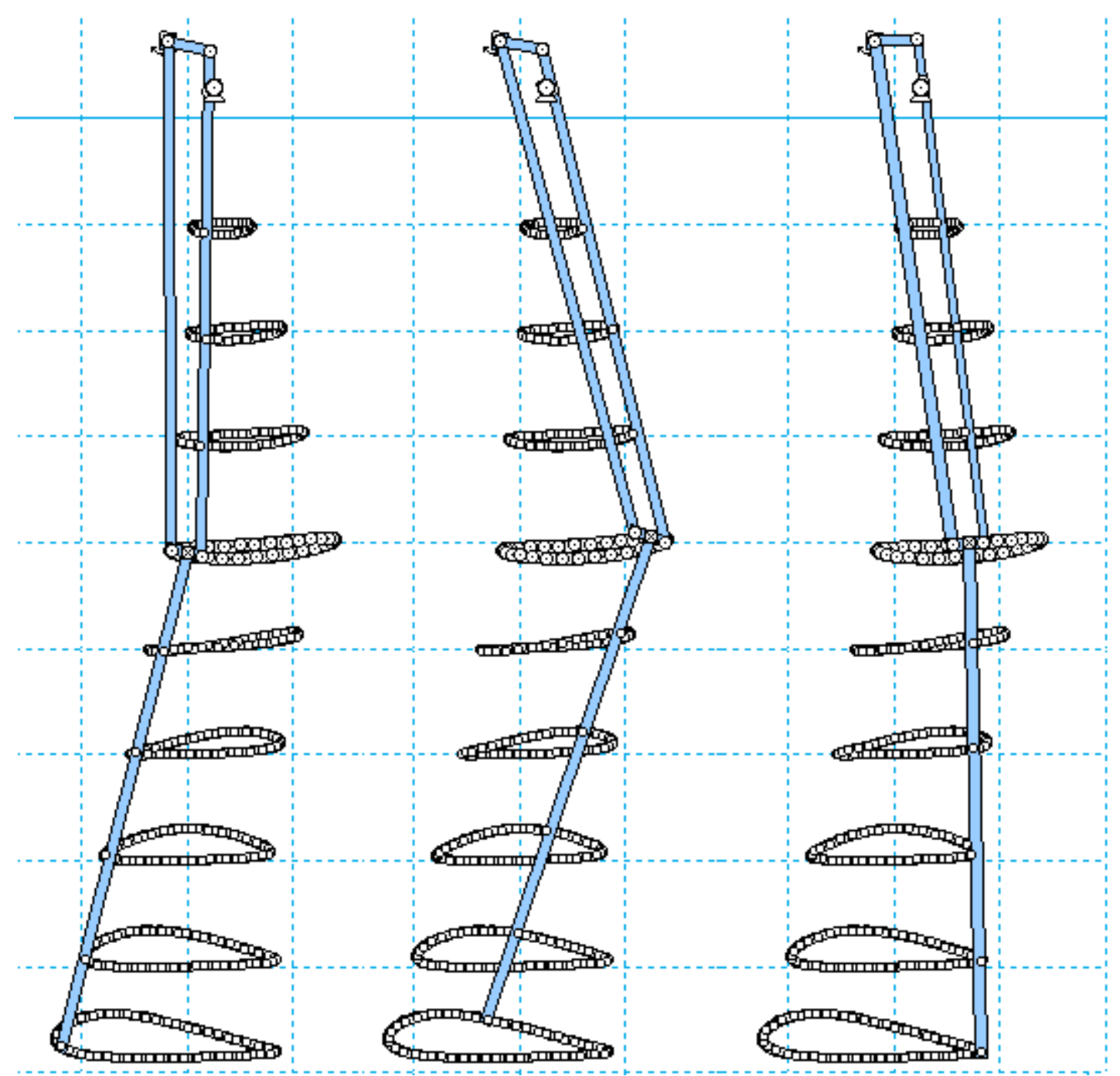

Figure 9. An alternative design of the proposed human-like leg mechanism created and tested using Working Model software package

\section{Summary and Conclusions}

The article presents a Stephenson III one degree-of-freedom (DOF) reproduction of the human walking apparatus. Qualitative synthesis namely to emulate the shape of the human leg is utilized to construct the profile of the mechanism. The resulting mechanism consists of a four-bar linkage and a dyad where the coupler point on the dyad represents the foot point. Type synthesis indicates that the resulting linkage is a Stephenson III mechanism. For the purpose of this study, link segments are selected such that the resulting mechanism both imitates the shape of the human leg. Furthermore, the path generated at the foot point is similar to that of the human walking gait. Alternative designs are generated and tested using Working Model software. The segments of the mechanism are iteratively tuned to arrive at the desired shape of the walking gait. The article provides alternative designs of the proposed leg mechanism. Moreover, we present position analysis of the proposed mechanism.

\section{References}

Batayneh, W., Al-Araidah, O., Mattson, C. A., \& Ismail-Yahaya, A. (2008). Design and Implementation of Human-Like Biped Walking Mechanism. The Third International Conference in Mechatronics, Kuala Lumpur, Malaysia.

Capi, G., Kaneko, S., Mitobe, K., Barolli, L., \& Nasu, Y. (2002). Optimal Trajectory Generation for a Prismatic Joint Biped Robot Using Genetic Algorithms. Robotics and Autonomous Systems, 38. 
Collins, S. H., \& Ruina, A. (2005). A Bipedal Walking Robot with Efficient and Human-like Gait. Proceedings of the IEEE International conference on robotics and automation, Barcelona, Spain.

Hirai, K., Hirose, M., Haikawa, Y., \& Takenaka, T. (1998). The development of honda humanoid robot. Proceedings of the IEEE International Conference on Robotics and Automation, Leuven, Belgium.

Hirose, M., Haikawa, Y., Takenaka, T., \& Hirai, K. (2001). Development of humanoid robot ASIMO, Proceedings of the IEEE/RSJ International Conference on Intelligent Robots and Systems, Maui, USA.

Hirukawa, H., Kanehiro, F., Kaneko, K., Kajita, S., Fujiwara, K., Kawai, Y., ... Inoue, H. (2004). Humanoid robotics platforms developed in HRP. Robotics and Autonomous Systems, 48(4). http://dx.doi.org/10.1016/j.robot.2004.07.007

Jun, S. K., \& Krovi, V. N. (2004). Design Considerations for an Articulated Leg-Wheel Locomotion Subsystem, Proceedings of IMECE. ASME International Mechanical Engineering congress and RD\&D Expo, Anaheim, California, USA.

Kaneko, K., Kanehiro, F., Kajita, S., Hirukawa, H., Kawasaki, T., Hirata, M., ... Isozumi, T. (2004). Humanoid robot HRP-2. Proceedings of the IEEE International Conference on Robotics and Automation, New Orleans, USA.

Kuroki, Y., Ishida, T., Yamagushi, J., Fujita, M., \& Doi, T. (2001). A small biped entertainment robot, Proceedings of the IEEE/RAS International Conference on Humanoid Robots.

Mehdigholi, H., \& Akbarnejad, S. (2008). Optimization of Watt's Six-bar Linkage to generate Straight and Parallel Leg Motion. Journal of Humanoids, $1(1)$.

Sangwan, V., Taneja, A., \& Mukherjee, S. (2004). Design of a Robust Self-Excited Biped Walking Mechanism. Mechanism and Machine Theory, 39.

Verrelst, B., Vanderborght, B., Vermeulen, J., Ham, R. V., Naudet, J., \& Lefeber, D. (2005). Control Architecture for the Pneumatically Actuated Dynamic Walking Biped "Lucy". Mechatronics, 15.

Weber, W., \& Weber, E. (1991). Mechanics of the Human Walking Apparatus. Springer-Verlag.

Yavin, Y. (2002). Modeling and Control of a Walking Four Link Robot. Mathematical and Computer Modeling, 35 .

\section{Copyrights}

Copyright for this article is retained by the author(s), with first publication rights granted to the journal.

This is an open-access article distributed under the terms and conditions of the Creative Commons Attribution license (http://creativecommons.org/licenses/by/3.0/). 